\title{
Biodegradable and Biocompatible Subcutaneous Implants Consisted of Ph-Sensitive Mebendazole- Loaded/Folic Acid-Targeted Chitosan Nanoparticles for Murine Triple-Negative Breast Cancer Treatment
}

\author{
Amirhosein Kefayat \\ Isfahan University of Medical Sciences \\ Maryam Hosseini \\ Amirkabir University of Technology \\ Fatemeh Ghahremani \\ Arak University of Medical Sciences \\ mohammad rafienia ( $\square$ m_rafienia@med.mui.ac.ir) \\ Isfahan University of Medical Sciences
}

\section{Research Article}

Keywords: Mebendazole, Chitosan nanoparticles, subcutaneous implants, Folic acid, Triple-negative breast cancer

Posted Date: July 30th, 2021

DOI: https://doi.org/10.21203/rs.3.rs-736440/v1

License: (c) (i) This work is licensed under a Creative Commons Attribution 4.0 International License.

Read Full License 


\section{Abstract}

Background: Mebendazole (MBZ) is a well-known anti-parasite drug with significant anti-cancer properties. However, MBZ exhibits low solubility, limited absorption efficacy, extensive first-pass effect, and low bioavailability. Therefore, multiple oral administration of high dose MBZ is required daily for achieving the therapeutic serum level which can cause severe side effects and patients' non-compliance.

Method: In the present study, MBZ-loaded/folic acid-targeted chitosan nanoparticles (CS-FA-MBZ) were synthesized, characterized, and used to form cylindrical subcutaneous implants for 4T1 triple-negative breast tumor (TNBC) treatment in BALB/c mice. The therapeutic efficacy of the CS-FA-MBZ implants was investigated after subcutaneous implantation in comparison with Control (no-treatment), MBZ (40 mg/ $\mathrm{kg}$, oral administration, twice a week for 2 weeks), and CS-FA implants, according to 4T1 tumors' growth progression, metastasis, and tumor-bearing mice survival time. Also, their biocompatibility was evaluated by blood biochemical analyzes and histopathological investigation of vital organs.

Results: The CS-FA-MBZ implants were completely degraded 15 days after implantation and caused about $73.3 \%, 46.5 \%, 37.3 \%$ decrease in the mean tumors' volume in comparison with the Control $\left(1050.5 \pm 120.7 \mathrm{~mm}^{3}\right), \operatorname{MBZ}\left(561.7 \pm 70.3 \mathrm{~mm}^{3}\right)$, and CS-FA $\left(658.3 \pm 88.1 \mathrm{~mm}^{3}\right)$ groups, respectively. Average liver metastatic colonies' number per microscope field at the CS-FA-MBZ (8.6 \pm 1.9$)$ group was significantly $(P<0.05)$ lower than the Control $(24.5 \pm 4.1)$, MBZ (14.1 \pm 2.5$)$, and CS-FA $(15.7 \pm 3.1)$ groups. In addition, the CS-FA-MBZ treated mice exhibited about $51 \%, 24 \%$, and $17 \%$ more survival time (days) after the cancer cells injection in comparison with the Control, MBZ, and CS-FA groups, respectively. Moreover, the CS-FA-MBZ implants were completely biocompatible based on histopathology and blood biochemical analyzes.

Conclusion: Taking together, CS-FA-MBZ implants were completely biodegradable and biocompatible with high therapeutic efficacy in a murine TNBC model.

\section{Background}

Breast cancer is the most common malignancy among women and the leading cause of cancer-related deaths in the female gender [1]. About $20 \%$ of all diagnosed breast cancers are categorized as triplenegative breast cancer (TNBC). This acronym simply means that the tumor doesn't express estrogen, progesterone, and human epidermal growth factor-2 receptors on its cells' surface [2]. TNBC is significantly more aggressive and invasive than other subtypes of breast cancer and exhibits higher relapse rates and shorter recurrence period [3]. Therefore, due to the absence of well-defined molecular targets, currently, the only therapeutic approach for TNBC patients is chemotherapy $[4,5]$. However, the outcome of chemotherapy with currently approved drugs isn't satisfying, and poor therapeutic response, severe side effects, and development of multidrug resistance are still big challenges in TNBC chemotherapy $[6,7]$. 
Mebendazole (MBZ) is a well-known antihelminthic drug, with high biocompatibility and low price which has been repurposed for anti-neoplastic treatment [8]. Many studies have reported anti-proliferative, proapoptotic, and anti-metastatic effects of MBZ on different cancer cell lines including chemoresistant cancer cell lines $[9,10]$. MBZ treatment causes a decrease or complete arrest of tumor growth, significant inhibition of tumor metastasis, and an increase of tumor-bearing mice survival time in different animal models of cancer [11-16]. Anti-neoplastic activities of MBZ can be attributed to inhibition of tubulin polymerization, decrease of tumor angiogenesis, blocking of pro-survival pathways, inhibition of matrix metalloproteinases function, and multi-drug resistance protein transporters activity [8, 17-19]. However, MBZ has limited bioavailability after oral administration as only $20 \%$ of the dosage reaches the systemic circulation. This can be related to the low solubility of MBZ, limited absorption efficacy, and extensive first-pass effect following oral administration [20,21]. Therefore, a high dose of MBZ is required for achieving the therapeutic serum level which can cause severe adverse effects and patients' noncompliance [22].

Drug-releasing implants have gained lots of attention for controlled drug release in the long-term treatments, especially cancer chemotherapy $[23,24]$. Implants that contain chemotherapy drugs have exhibited many advantages over intravenous or oral drug administration routes including the elimination of daily multiple injections and maintenance of the steady-state concentration of the drug [25-29]. Biodegradable polymers are the most utilized materials for developing these implants [30]. Chitosan (CS) is a well-known biodegradable biopolyaminosaccharide with a natural origin. The chitosan-based drug carriers and implants exhibit high biodegradability, low toxicity, and appropriate biocompatibility. In addition, chitosan per se has considerable anti-proliferative, pro-apoptotic, anti-angiogenetic, and antimetastatic effects on different types of tumors [31-33]. Different forms of chitosan-based drug delivery systems have been used and chitosan nanoparticles are one of the most advanced ones for the enhancement of anti-cancer drugs efficacy. They can be defined as spherical, biocompatible, and biodegradable nanostructures with high drug-loading efficacy [34-36]. Chitosan nanoparticles can be used for controlled release of different types of drugs and enhancement of tumor drug delivery efficacy [37-41]. In addition, chitosan nanoparticles decorating with targeting ligands that can bind to the malignant cells' surface receptors have received lots of attention for enhancement of tumor drug delivery efficacy $[42,43]$. One of the most efficient targeting agents for cancer cells specific targeting is folic acid (FA) due to overexpression of the folate receptor on their membrane in comparison with normal cells. FA has many advantages over other targeting agents, including small molecular weight, simple chemical properties, receptor-medicated endocytosis of the folic acid decorated-carriers, extremely low immunogenicity, overexpression on cancer cells membrane, and limited expression on normal cells' surface [44-47]. Although TNBC cells are known due to the lack of different molecular targets on their membrane, FA is a highly overexpressed receptor on their surface [48-50]. Therefore, FA-modified drug delivery systems can increase the drug concentration at the TNBC tumors site and decline its side effects at normal tissues [51].

In the present study, MBZ-loaded/folic acid-targeted chitosan nanoparticles (CS-FA-MBZ) were used to form cylindrical implants for $4 \mathrm{~T} 1$ triple-negative breast tumor treatment in BALB/c mice. The therapeutic 
efficacy of the CS-FA-MBZ implants was investigated after subcutaneous implantation in comparison with Control, MBZ (40 mg/kg, oral administration, twice a week for 2 weeks), and CS-FA implants according to 4T1 tumors' growth progression, metastasis, and tumor-bearing mice survival time. Also, the CS-FA-MBZ implant's biocompatibility was evaluated by blood biochemical analyzes and vital organs histopathological investigations. In this study, 4T1 murine triple-negative mammary carcinoma was used as an experimental animal model with high similarity to human TNBC. This cell line is a highly tumorigenic and invasive cancer cell line that can spontaneously metastasize from the primary tumor in the mammary gland to multiple distant sites. Also, its metastasis pattern is very similar to that of human breast cancer [52-55].

\section{Materials And Methods}

\subsection{Material}

Medium-molecular weight chitosan (190 to 310 kDa, MMW), Sodium tripolyphosphate (TPP), 1-ethyl-3-(3dimethylaminopropyl) carbodiimide (EDC), N-hydroxysuccinimide (NHS), folic acid (97\%, FA), Tween-80 (a non-ionic surfactant), mebendazole (MBZ), ammonia (25\%, $\left.\mathrm{NH}_{4} \mathrm{OH}\right)$, acetic acid, dimethyl sulfoxide (DMSO), phosphate-buffered saline powder ( $\mathrm{pH}=7.4 \mathrm{PBS}$ ), methanol and acetone were purchased from Sigma-Aldrich and used without further purification unless stated otherwise. RPMI 1640, fetal bovine serum (FBS), phosphate buffer saline (PBS), penicillin/streptomycin, ethanol $(96 \%, \mathrm{v} / \mathrm{v})$, trypsin-EDTA (0.25\%) were purchased from Merck (Germany).

\subsection{Conjugation of folic acid to chitosan (CS-FA preparation)}

CS-FA was fabricated as reported in the literature [56]. Briefly, $0.5 \mathrm{~g}$ of FA and $0.2 \mathrm{~g}$ of EDC were initially dissolved in anhydrous DMSO $(20 \mathrm{~mL})$ under constant stirring at room temperature $(2 \mathrm{~h})$. Then, the solution was dropped into the CS solution $0.5 \%(\mathrm{w} / \mathrm{v})$ prepared in acetate buffer $(0.1 \mathrm{M}, \mathrm{pH} 4.7)$ and at room temperature in dark for $16 \mathrm{~h}$. Thereafter, the $\mathrm{pH}$ of the solution was adjusted to 9.0 by the addition of $\mathrm{NaOH}(1.0 \mathrm{M})$. The resulting precipitate was collected by centrifugation, then purified by dialysis against phosphate-buffered saline (PBS, $\mathrm{pH} 7.4$ ) for 2 days and against water for another 4 days. Finally, yellow-colored CS-FA products were collected and freeze-dried.

\subsection{Preparation of the CS-FA-MBZ nanoparticles}

CS-FA-MBZ nanoparticles were synthesized according to the previously reported method with some modifications [57]. Initially, CS-FA (0.1 g) was dissolved in a solution containing acetic acid ( $1 \% \mathrm{v} / \mathrm{v}, 20$ $\mathrm{mL})$, then left under stirring at room temperature in dark for $16 \mathrm{~h}$ to prepare a solution of CS-FA $(0.5 \%$ $\mathrm{w} / \mathrm{v})$. The $\mathrm{pH}$ of the solution was adjusted to 4.8 by the addition of $\mathrm{NaOH}(1.0 \mathrm{M})$. Afterward, $250 \mu \mathrm{L}$ of Tween- 80 was added dropwise and left for $2 \mathrm{~h}$ under stirring at $45^{\circ} \mathrm{C}$. In the next step, $0.01 \mathrm{~g} \mathrm{MBZ}$ was dissolved in $0.5 \mathrm{M}$ methanolic hydrochloride [58] and then added to the former solution and stirred for 30 min. Finally, $10 \mathrm{~mL}$ TPP aqueous solution $(0.5 \% \mathrm{w} / \mathrm{v})$ was dropped to the CS-FA solution slowly under 
magnetic stirring (800 rpm) at room temperature for $1 \mathrm{~h}$ then the nanoparticles were collected by centrifugation (12000 rpm, $30 \mathrm{~min}$ ). The resulting CS-FA-MBZ nanoparticles were lyophilized and stored.

\subsection{MBZ loading}

MBZ was loaded during the formation of CS-FA nanoparticles as reported in the literature [57]. To calculate MBZ encapsulation efficacy (EE\%) and loading capacity (LC\%), unloaded MBZ content in the supernatant of the last step was determined through a calibration curve of MBZ standard solution by UV-Visible spectroscopy at $234 \mathrm{~nm}$ [58]. The MBZ loading ratio of the nanoparticles was calculated by the following equations ( 1 and 2 ):

MBZ encapsulation efficacy $(E E \%)=\frac{\text { Mass of the loaded MBZ }}{\text { Mass of the initial MBZ }} \times 100$

MBZ loading capacity (LC\%) $=\frac{\text { Mass of the loaded MBZ }}{\text { Mass of the final product }} \times 100$

\subsection{In vitro drug release pattern from the CS-FA-MBZ nanoparticles}

To evaluate the release behavior of MBZ from CS-FA-MBZ nanoparticles, $5 \mathrm{mg}$ of the CS-FA-MBZ nanoparticles were immersed in PBS solution containing Tween-80 $(0.1 \% \mathrm{w} / \mathrm{v})$ at $\mathrm{pH}$ values of $5.5,6.8$, and 7.4. The release profile was assessed at $37^{\circ} \mathrm{C}$ in dark under shaking at $100 \mathrm{rpm}$ for up to 1 week [59]. At each predetermined time interval, the nanoparticles were centrifuged (10,000 rpm for $15 \mathrm{~min})$ and the released medium was collected and replaced with equivalent fresh PBS solution. The cumulative percentage of released MBZ was determined by UV-Visible spectrophotometry at $234 \mathrm{~nm}$.

\subsection{Nanoparticles characterization and implants fabrication}

To assess the structure and interaction of CS, CS-FA, and CS-FA-MBZ, Fourier Transform Infrared Spectroscopy (FTIR) was used by a Bruker Equinox 55 spectrometer with the $\mathrm{KBr}$ pellets method. To evaluate the size and morphology of nanoparticles, scanning electron microscopy (SEM) was acquired using FEI ESEM QUANTA 200, MIRAll, and MIRAIII Tescan. The average size and size distribution of particles were determined by measuring the diameter of 100 particles of SEM images using ImageJ software. The ultraviolet-visible (UV-vis) spectra were recorded by a PerkinElmer Lambda 950 spectrophotometer (wavelength range: $200-800 \mathrm{~nm}$ ). The zeta potential, size distribution, and polydispersity of the prepared nanoparticles were measured by Dynamic Light Scattering (DLS) (Malvern Instruments). A well-dispersed aqueous suspension of the prepared nanoparticles was applied. Each experiment was carried out in triplicate and data were presented as means \pm standard deviations. For fabricating an implant, adequate mass of the synthesized CS-FA-MBZ nanoparticles (according to the mouse body weight and its needed dosage of MBZ) were pressed in a steel die at 1500 psi to from cylindric implants (usually $6 \mathrm{~mm}$ diameters and $3 \mathrm{~mm}$ height). 


\subsection{Animals ethics, care, and handling}

All animal experiments complied with the ARRIVE guidelines and were conducted according to the guidelines of the European Communities Council Directive (2010/63/UE) and the Isfahan University of Medical Sciences for the care and use of laboratory animals. Likewise, all the procedures, protocols, and steps were approved by the ethics committee of the Isfahan University of Medical Sciences (IR.MUI.RESEARCH.REC.1399.125). Female BALB/c mice (weight: $25 \pm 2 \mathrm{~g}$ ) were purchased from the Pasteur Institute of Tehran, Iran. The mice were acclimatized to the laboratory environment $\left(24 \pm 2{ }^{\circ} \mathrm{C}\right.$ temperature, $50 \pm 10 \%$ relative humidity, and $12 \mathrm{~h}$ light/ $12 \mathrm{~h}$ dark cycles) for 14 days before involving into the experiments. All mice were fed sterilized standard mouse chow and water ad libitum. Overdose of Ketamine-Xylazine (KX) solution through intraperitoneal injection was used for the mice sacrifice.

\subsection{Tumor implantation}

4T1 cancer cells (murine mammary carcinoma) were purchased from the Pastor Institute of Tehran, Iran. The cells were cultured in RPMI 1640 medium containing 10\% fetal bovine serum (FBS). The cells were incubated at $37{ }^{\circ} \mathrm{C}$ in a humidified incubator in $5 \% \mathrm{CO}_{2}$ atmosphere. When the cells reached adequate numbers, were harvested from culture flasks by trypsin and washed three times with PBS. The mice were injected with $2 \times 10^{6}$ cells suspended in $50 \mu \mathrm{L}$ of FBS-free DMEM-F12, subcutaneously (s.c.) into the left 4th abdominal mammary fat pad.

\subsection{Tumor-bearing mice grouping and therapeutic approaches}

For this experiment 32 tumor-bearing mice were used. When the tumors' volume reached $50-70 \mathrm{~mm}^{3}(3 \mathrm{rd}$ day after the cancer cells injection), mice were divided into four groups $(n=8)$ including (1) Control, (2) MBZ, (3) CS-FA implants, (4) CS-FA-MBZ implants. The tumor-bearing mice in the 2 nd group were treated with oral administration )p.o.( of MBZ (40 mg/kg, twice a week for 2 weeks according to previous studies [14]) from the 3rd day after the cancer cells injection. In the 3rd and 4th groups, the tumor-bearing mice were anesthetized with intraperitoneally injection of Ketamine-Xylazine (KX) solution (Ketamine: 100 $\mathrm{mg} / \mathrm{kg}$, Xylazine: $10 \mathrm{mg} / \mathrm{kg}$ ). The left flank was shaved and scrubbed with betadine. The scrub solution was wiped away from the surgical site with alcohol $70 \%$ and covered with a sterile drape. Then, a small $(\sim 1 \mathrm{~cm})$ incision was made and the implant was embedded under sterile conditions, and the skin was stitched with nylon (4 - 0). All the operations were done under complete anesthesia. To manage postsurgical pain, ketoprofen $(5 \mathrm{mg} / \mathrm{kg}$ ) was administered subcutaneously until the next $72 \mathrm{~h}$. The mice were monitored daily for prolonged signs of pain, weight loss, or surgical site infections. If any signs of pain, wounds infection, massive necrosis, and hemorrhage, diffuse metastasis were observed during any steps of the study, the mice were sacrificed by KX overdose. In the Control group, one incision was made at the left flank of the tumor-bearing mice and sutured without implantation of any implants. To determine tumors' growth progression, the greatest longitudinal diameter (length) and the greatest transverse diameter (width) of the tumors were measured every 3 days until the 18th after cancer cells injection. 
Then, the tumor's volume was calculated by the tumor volume Eq. (3). For survival analysis, the tumorbearing mice were observed for 70 days after treatment administration. The animals' death was recorded every day. It should be mentioned that standardized humane endpoints based on the current guidelines for endpoints in animal tumor studies were used [60-62].

$$
\text { Tumor volume }=\frac{(\text { Tumor length }) \times(\text { Tumor width })^{2}}{2}
$$

\subsection{T1 breast tumors' metastasis}

For this experiment, 20 tumor-bearing mice were involved $(n=5)$ and the groups and therapeutic methods were completely the same as the previous section. The mice were sacrificed by overdose of ketamine/xylazine 30 days after cancer cell implantation and their livers were harvested and fixed in $10 \%$ neutral buffered formalin solution. An automatic tissue processor (Sakura, Japan) was employed to process the fixed samples. Then, a microtome (Leica Biosystems, Germany) was utilized to cut $4 \mu \mathrm{m}$ thickness serial sections from the paraffin-embedded blocks. The sections were stained with Hematoxylin \& Eosin (H\&E) staining protocol according to previous studies [55, 63]. A minimum of 10 random microscopic fields was observed under the $10 \times$ objective lens of a light microscope (Olympus, Japan) to report the mean number of metastatic colonies per microscopic field of the liver. Furthermore, the occupied area by metastatic colonies in each microscopic field of the liver (magnification $\times 100$ ) was quantified by the Qupath software. The mean percentage of occupied space by liver metastatic colonies in each microscopic field was reported for each sample.

\subsection{Histopathology and blood biochemical assays}

For evaluating the safety of the subcutaneous CS-FA-MBZ implants, 10 healthy mice were involved and randomly divided into two groups $(n=5)$ including (1) Control and (2) CS-FA-MBZ implants. After CS-FAMBZ implantation, the mice were monitored for general appearance and behavioral parameters for 30 days. They were under close monitored for any signs of toxicity and behavioral changes including weakness, salivation, anorexia, diarrhea, aggressiveness, eyes and ears discharge, noisy breathing, activity, convulsion, cachexia, pain, or any signs of illness in each group for 30 days [64]. On the 30th day, the mice were sacrificed and blood urea nitrogen (BUN), creatinine $(\mathrm{Cr})$, alanine aminotransferase (ALT), and aspartate aminotransferase (AST) levels were measured in the discarded serums [65]. In addition, lungs, kidneys, liver, and spleen were harvested and fixed, processed, and H\&E stained. Histological photographs were obtained using a digital light microscope (Olympus, Japan).

\subsection{Statistical Analysis}

The statistical analyzes were performed using one-way analysis of variance (ANOVA) with Tukey's posthoc test by JMP 14.0 software (SAS Institute, Japan). The results were statistically significant at $P<0.05$ (*: $P \bigotimes 0.05$, ns: not significant). All values were expressed as the mean \pm standard deviation.

\section{Results And Discussion}




\subsection{Fabrication and characterization of the CS-FA-MBZ nanoparticles}

For fabrication of the CS-FA-MBZ nanoparticles, at first, folic acid was conjugated to chitosan in the presence of EDC as carboxyl activating agent to produce CS-FA (Figure S1). The activated carboxyl moiety of FA was covalently linked to amine groups of CS [66]. In the next step, the cross-linking reaction between CS-FA and TPP lead to the formation of nanoparticles in which the negatively charged TPP was electrostatically adsorbed to positively charged free protonated amine groups of CS-FA and MBZ was encapsulated during the synthesis process (Figure 1). The successful introduction of folate to CS chains was evaluated by FTIR analysis. The FTIR spectra of CS, FA, and CS-FA were shown in Figure S2. The characteristic bands of CS located at $3422 \mathrm{~cm}^{-1}$ assigned to $\mathrm{O}-\mathrm{H}$ stretching vibration overlapped with $\mathrm{N}-\mathrm{H}$ stretching mode. The bands observed at 2920 and $2880 \mathrm{~cm}^{-1}$ were attributed to the $\mathrm{C}-\mathrm{H}$ stretching vibrations of CS. Moreover, the peaks that appeared at 1656 and $1605 \mathrm{~cm}^{-1}$ corresponded to the C-O stretching vibration of amide I and $\mathrm{N}-\mathrm{H}$ bending vibration of amide II, respectively [67]. FTIR spectrum of FA showed characteristic bands located at 1696,1607 , and $1486 \mathrm{~cm}^{-1}$ related to the $\mathrm{C}=0$ amide stretching vibration of the carboxyl group, $\mathrm{N}-\mathrm{H}$ bending vibration of $\mathrm{CONH}$, and the stretching vibration of $\mathrm{C}=\mathrm{C}$ of the phenyl ring of FA, respectively [68]. In the CS-FA spectrum, the absorption peaks at 1635 and $1031 \mathrm{~cm}^{-1}$ could be attributed to the vibration of C-N [67]. The amid band at $1656 \mathrm{~cm}^{-1}$ of CS shifts to $1635 \mathrm{~cm}^{-1}$ because of overlap with the newly formed amide bond, and also a new N-H bending vibration located at $1520 \mathrm{~cm}^{-1}$ confirmed the successful coupling of FA to CS [69]. After the formation of the CS-FAMBZ nanoparticles, FTIR analysis was carried out to find the drug-polymer compatibility (Figure 2). The characteristic absorption peaks of pure MBZ at $3403,1717,1647$, and $1523 \mathrm{~cm}^{-1}$ were also observed for CS-FA-MBZ. The stretching vibration of amide I of the carbamate group of MBZ $\left(1717 \mathrm{~cm}^{-1}\right)$ was also observed at the CS-FA-MBZ nanoparticles spectrum in the same location [70]. The $\mathrm{N}-\mathrm{H}$ stretching and $\mathrm{CNH}$ vibration were observed at 3403 and $1523 \mathrm{~cm}^{-1}$ for MBZ, 3405, and $1525 \mathrm{~cm}^{-1}$ for CS-FA-MBZ. Hence, no interaction was observed from FTIR detection.

Figures $3 a$ and $b$ show SEM images of the CS-FA-MBZ nanoparticles at different magnifications, in which the nanoparticles were uniform and exhibited a spherical morphology. The corresponding particle size distribution histograms (dry state) were obtained by measuring the size of 100 nanoparticles in SEM images by the "Image J" software (Figure 3c). A narrow size distribution with a mean size of $153.3 \pm 18.4$ $\mathrm{nm}$ was measured. Moreover, the particle size distribution of the CS-FA-MBZ was determined by DLS measurement (wet state) and the mean size (diameter) of the nanoparticles was measured $182 \pm 12.1$ $\mathrm{nm}$ with a low polydispersity index ( $\mathrm{PDI}<0.2)$, illustrating a narrow size distribution and confirmed the result obtained from SEM as well (Figure 3d). Due to the measuring of hydrodynamic radius, the DLS analysis showed a slightly larger size for the nanoparticles as compared to SEM photographs [71]. The zeta potential values showed that the positive charge of chitosan due to the presence of the amine groups decreased after FA conjugation (Figure 2e). It could be related to the interaction of FA molecules with these amine groups of CS, leading to the neutralization of the potential value [72]. Additionally, the results depicted that the encapsulation of MBZ into the CS-FA nanoparticles could not induce a noticeable zeta value change in the final nanoparticles with the preserved positive value of $+27 \mathrm{mV}$. 


\subsection{MBZ loading}

MBZ, as a hydrophobic drug, was loaded into the CS-FA nanoparticles. The UV-Vis spectrum of MBZ (Figure 4a) showed the characteristic absorption band at $234 \mathrm{~nm}$ which also could be observed for CSMBZ. This band along with the absorption band of FA at $280 \mathrm{~nm}$ with a slight shift to longer wavelengths could be observed in the spectrum of CS-FA-MBZ, indicating the appropriate MBZ loading into the CS-FA nanoparticles. The encapsulation efficacy (EE) and loading capacity (LC) of MBZ were calculated by plotting the standard calibration curve with a linear curve fit equation (Figure S3.). The value of EE and LC were achieved $57.7 \%$ and $10.5 \%$ respectively, demonstrating the valuable capability of the target system for MBZ loading.

\subsection{In vitro $\mathrm{MBZ}$ release study}

The release behavior of MBZ from the CS-FA-MBZ nanoparticles was evaluated at different $\mathrm{pH}$ values of $5.5,6.8$, and 7.4 to mimic tumor microenvironment, physiological conditions ( $\mathrm{pH}=7.4$ like bloodstream) and endocytic compartments conditions $(\mathrm{pH}=5.5)$ [59]. As Figure $4 \mathrm{~b}$ illustrates, the CS-FA-MBZ nanoparticles exhibited a continuous and sustained release profile at different $\mathrm{pH}$ values started with a burst release within $6 \mathrm{~h}$ followed by a gradual release up to 1 week. The initial fast release could be related to the absorbed MBZ on the surface of nanoparticles [68]. Moreover, a pH-responsive behavior of MBZ was observed. After 1 week, the release of MBZ was around $62 \%, 49 \%$, and $38 \%$ at pH values of $5.5,6.8$, and 7.4 , respectively. This behavior could be assigned to the high swelling ability of CS which was induced by protonation of the amine groups of the polymer in an acidic environment [68]. Considering the acidic microenvironment of cancerous tissues and intracellular organelles such as endosomes and lysosomes, the $\mathrm{pH}$-sensitive behavior of the CS-FA-MBZ nanoparticles improves their capability for tumor-specific drug delivery and reduces the probable adverse effects to normal tissues.

\subsection{The CS-FA-MBZ implants effect on the 4T1 breast tumors' growth progression}

As Figure 5a illustrates, the CS-FA and CS-FA-MBZ implants were s.c. implanted in the left flank of the tumor-bearing mice inside a small incision on the $3^{\text {rd }}$ day after the cancer cell injection. The implants were completely palpable even after suturing the incision. The implants completely degraded until the $18^{\text {th }}$ day in both CS-FA and CS-FA-MBZ groups and nothing was palpable at the implantation site. This means that the implants were dissociated and release their composing agents means CS-FA-MBZ nanoparticle.

The therapeutic effect of the CS-FA-MBZ implants on inhibition of 4T1 tumors' growth progression was evaluated by serial measurement of tumor's diameters and compared with the Control, MBZ $(40 \mathrm{mg} / \mathrm{kg}$, oral administration, twice a week for two weeks), and CS-FA groups (Figure 5b). All treatments were initiated from the $3^{\text {rd }}$ day after cancer cells injection. As Figure $5 \mathrm{~b}$ illustrates, the CS-FA-MBZ implants could significantly inhibit the breast tumors growth progression in comparison with all other groups. On the last day of tumors' volume monitoring ( $18^{\text {th }}$ day after cancer cells injection), the mean tumors' 
volume at the CS-FA-MBZ group $\left(658.3 \pm 88.1 \mathrm{~mm}^{3}\right)$ was significantly $(P<0.05)$ lower than the Control $\left(1050.5 \pm 120.7 \mathrm{~mm}^{3}\right), \mathrm{MBZ}\left(561.7 \pm 70.3 \mathrm{~mm}^{3}\right)$ and CS-FA $\left(658.3 \pm 88.1 \mathrm{~mm}^{3}\right)$ groups. The CS-FA-MBZ implants could cause about $73.3 \%, 46.5 \%, 37.3 \%$ decrease in the mean tumors' volume in comparison with the Control, MBZ, and CS-FA groups, respectively. Therefore, CS-FA-MBZ implants exhibit high efficacy in inhibiting breast tumors growth.

On the $6^{\text {th }}$ and $18^{\text {th }}$ days ( $3^{\text {rd }}$ and $15^{\text {th }}$ days after surgery, respectively), some implants-bearing mice were sacrificed to observe what is happening on the CS-FA and CS-FA-MBZ implants' site (Figure 5c). As illustrated in Figure $5 \mathrm{c}$, the implants were surrounded by a thin transparent membrane on the $6^{\text {th }}$ day. On the $18^{\text {th }}$ day, the implants were completely degraded and disappeared. As Figure 6 illustrates, histopathological evaluations of the implantation site on the $6^{\text {th }}$ day demonstrated that the surrounding membrane consisted of connective tissue. Also, limited mononuclear cells infiltration was observed at the implants' bed (Figure 6).

\subsection{The CS-FA-MBZ implants effect on metastasis and tumor-bearing mice survival time}

Metastasis is the main cause of cancer-related deaths. The formation of metastatic colonies at vital organs like the liver disrupts their function and causes organ failure [73]. Therefore, to evaluate the effect of the CS-FA-MBZ implants on metastasis formation, H\&E sections were used to count the metastatic colonies at tumor-bearing mice liver after 35 days from cancer cells injection (Figure 7). Histopathological evaluations demonstrated significant inhibition of the liver metastatic colonies formation at the CS-FAMBZ $(8.6 \pm 1.9)$ treated mice in comparison with the Control $(24.5 \pm 4.1)$, MBZ $(14.1 \pm 2.5)$, and CS-FA $(15.7 \pm 3.1)$ groups (Figure $7 a$ and $b)$. Besides, the metastatic colonies occupied significantly $(P<0.05)$ lower space in the liver sections (per microscopic field) of the CS-FA-MBZ treated group in comparison with the other groups (Figure 7c). In addition, the CS-FA-MBZ group exhibited about 51\%, 24\%, and 17\% more survival time (days) in comparison with the Control, MBZ, and CS-FA groups, respectively (Figure $7 d$ ). This increase in the tumor-bearing mice survival time can be attributed to significant inhibition of $4 \mathrm{~T} 1$ tumors' growth and metastasis.

\section{Biocompatibility of the subcutaneous CS-FA-MBZ implants}

The safety and biocompatibility of implants are very important for clinical application. Therefore, the CSFA-MBZ implants were subcutaneously implanted in non-tumor-bearing mice. Then, the implant-bearing mice were exactly monitored according to general appearance and behavioral parameters, blood biochemical analyzes, and histopathological evaluation of vital organs. No sign of change in the mice's appearance, behavioral pattern, and food intake were observed during the 30 days (Tables $1 S$ ). On the $30^{\text {th }}$ day, the animals were sacrificed and their plasma was collected for biochemical (Figure 8a) analyses and the vital organs were harvested for histopathological exams (Figure 8b). No sign of organ damage was observed in either H\&E sections and blood biochemical analyzes. Chitosan nanoparticles are the main component of CS-FA-MBZ implants. Chitosan is a natural biodegradable biopolymer. The enzymatic degradation of chitosan causes its transformation to some components which are completely safe. 
Many enzymes have the ability to degrade chitosan and the most well-known one is lysozyme as a nonspecific protease. This enzyme which presents in all mammalian tissues and fluids, plays a key role in degradation of chitosan-based implants in vivo. It targets the acetylated residues of chitosan polymer and degrades chitosan to non-toxic oligosaccharides which can be excreted or incorporated to glycosaminoglycans and glycoproteins [30,74-76]. Also, eight human chitinases (in the glycoside hydrolase 18 family) have been identified, three of which have shown enzymatic activity [77].

\section{Conclusion}

Chemotherapy drugs loaded implants have exhibited higher efficacy in comparison with common routes of drug administration. In the present study, folic acid-targeted chitosan nanoparticles were used as a carrier to increase MBZ therapeutic efficacy in the triple-negative breast cancer-bearing BALB/c model. A definite amount of MBZ-loaded CS-FA nanoparticles were compressed to form cylindrical CS-FA-MBZ implants which were subcutaneously implanted at mice's flank. The CS-FA-MBZ implants could significantly inhibit 4T1 breast tumor growth and metastasis. Therefore, these biodegradable and biocompatible implants can be an appropriate choice for further experiments in breast cancer and other cancers treatment

\section{Abbreviations}

Triple-negative breast cancer: TNBC

Mebendazole: MBZ

Folic acid: FA

Chitosan: CS

KX: Ketamine-Xylazine

\section{Declarations}

\section{Ethics approval and consent to participate}

All animal experiments complied with the ARRIVE guidelines and were conducted according to the guidelines of the European Communities Council Directive (2010/63/UE) and the Isfahan University of Medical Sciences for the care and use of laboratory animals. Likewise, all the procedures, protocols, and steps were approved by the ethics committee of the Isfahan University of Medical Sciences (IR.MUI.RESEARCH.REC.1399.125).

\section{Consent for publication}

All authors have read and agreed to publish this manuscript. 


\section{Availability of data and material}

All datasets are available upon reasonable request.

\section{Competing interests}

The authors declared no potential conflicts of interest with respect to the research, authorship, and/or publication of this article.

\section{Funding}

This research was supported by Isfahan University of Medical Sciences.

\section{Authors' contributions}

The study was designed by A. Kefayat. In vivo assessments were carried out by A. Kefayat, F. Ghahremani, and M. Rafienia. The nanoparticles synthesis and characterization were done by M. Hosseini. The manuscript writing and revisions were done by A. Kefayat, M. Hosseini, F. Ghahremani, and M. Rafienia. All the authors read and approved the manuscript before submission.

\section{References}

1. DeSantis, C.E., et al., Breast cancer statistics, 2017, racial disparity in mortality by state. 2017. 67(6): p. 439-448.

2. Perou, C.M., et al., Molecular portraits of human breast tumours. 2000. 406(6797): p. 747-752.

3. Kumar, P., R.J.A.o.g. Aggarwal, and obstetrics, An overview of triple-negative breast cancer. 2016. 293(2): p. 247-269.

4. Wang, J., et al., Adjuvant chemotherapy and radiotherapy in triple-negative breast carcinoma: a prospective randomized controlled multi-center trial. 2011. 100(2): p. 200-204.

5. Sorolla, A., et al., Triple-hit therapeutic approach for triple negative breast cancers using docetaxel nanoparticles, EN1-iPeps and RGD peptides. 2019. 20: p. 102003.

6. Al-Mahmood, S., et al., Metastatic and triple-negative breast cancer: challenges and treatment options. 2018. 8(5): p. 1483-1507.

7. Nedeljković, M. and A.J.C. Damjanović, Mechanisms of Chemotherapy Resistance in Triple-Negative Breast Cancer-How We Can Rise to the Challenge. 2019. 8(9): p. 957.

8. Guerini, A.E., et al., Mebendazole as a candidate for drug repurposing in oncology: an extensive review of current literature. 2019. 11(9): p. 1284.

9. Coyne, C., et al., Gemcitabine-(C4-amide)-[anti-HER2/neu] anti-neoplastic cytotoxicity in dual combination with mebendazole against chemotherapeutic-resistant mammary adenocarcinoma. 2013. 2(2). 
10. Doudican, N., et al., Mebendazole induces apoptosis via Bcl-2 inactivation in chemoresistant melanoma cells. 2008. 6(8): p. 1308-1315.

11. Larsen, A.R., et al., Repurposing the antihelmintic mebendazole as a hedgehog inhibitor. 2015. 14(1): p. 3-13.

12. Bai, R.-Y., et al., Brain penetration and efficacy of different mebendazole polymorphs in a mouse brain tumor model. 2015. 21(15): p. 3462-3470.

13. He, L., et al., Mebendazole exhibits potent anti-leukemia activity on acute myeloid leukemia. 2018. 369(1): p. 61-68.

14. Mukhopadhyay, T., et al., Mebendazole elicits a potent antitumor effect on human cancer cell lines both in vitro and in vivo. 2002. 8(9): p. 2963-2969.

15. Pantziarka, P., et al., Repurposing Drugs in Oncology (ReDO)-mebendazole as an anti-cancer agent. 2014. 8.

16. Martarelli, D., et al., Mebendazole inhibits growth of human adrenocortical carcinoma cell lines implanted in nude mice. 2008. 61(5): p. 809-817.

17. Pinto, L.C., et al., Mebendazole, an antiparasitic drug, inhibits drug transporters expression in preclinical model of gastric peritoneal carcinomatosis. 2017. 43: p. 87-91.

18. Sung, S.J., et al., Autophagy is a potential target for enhancing the anti-angiogenic effect of mebendazole in endothelial cells. 2019. 27(1): p. 117.

19. Bai, R.-Y., et al., Effective treatment of diverse medulloblastoma models with mebendazole and its impact on tumor angiogenesis. 2015. 17(4): p. 545-554.

20. Dawson, M., R. Allan, and T.J.B.j.o.c.p. Watson, The pharmacokinetics and bioavailability of mebendazole in man: a pilot study using [3H]-mebendazole. 1982. 14(3): p. 453-455.

21. Dawson, M., et al., The pharmacokinetics and bioavailability of a tracer dose of [3H]-mebendazole in man. 1985. 19(1): p. 79-86.

22. de la Torre-Iglesias, P.M., et al., Enhanced bioavailability and anthelmintic efficacy of mebendazole in redispersible microparticles with low-substituted hydroxypropylcellulose. 2014. 8: p. 1467.

23. Belz, J.E., et al., Sustained Release Talazoparib Implants for Localized Treatment of BRCA1-deficient Breast Cancer. Theranostics, 2017. 7(17): p. 4340.

24. Park, C.-W., et al., In vitro/in vivo evaluation of NCDS-micro-fabricated biodegradable implant. 2010. 33(3): p. 427-432.

25. Arias, J.L., Novel strategies to improve the anticancer action of 5-fluorouracil by using drug delivery systems. Molecules, 2008. 13(10): p. 2340-2369.

26. Weinberg, B.D., E. Blanco, and J. Gao, Polymer implants for intratumoral drug delivery and cancer therapy. Journal of pharmaceutical sciences, 2008. 97(5): p. 1681-1702.

27. Talebian, S., et al., Biopolymers for antitumor implantable drug delivery systems: recent advances and future outlook. 2018. 30(31): p. 1706665. 
28. Zhang, Z., et al., Sandwich-Like Fibers/Sponge Composite Combining Chemotherapy and Hemostasis for Efficient Postoperative Prevention of Tumor Recurrence and Metastasis. 2018. 30(49): p. 1803217.

29. Kuang, G., et al., Biphasic drug release from electrospun polyblend nanofibers for optimized local cancer treatment. 2018. 6(2): p. 324-331.

30. Kefayat, A. and S.J.I.j.o.b.m. Vaezifar, Biodegradable PLGA implants containing doxorubicin-loaded chitosan nanoparticles for treatment of breast tumor-bearing mice. 2019. 136: p. 48-56.

31. Nam, K.-S., Y.-H.J.J.o.m. Shon, and biotechnology, Suppression of metastasis of human breast cancer cells by chitosan oligosaccharides. 2009. 19(6): p. 629-633.

32. Li, Y., et al., Chitosan sulfate inhibits angiogenesis via blocking the VEGF/VEGFR2 pathway and suppresses tumor growth in vivo. 2019. 7(4): p. 1584-1597.

33. Adhikari, H.S. and P.N.J.I.J.o.B. Yadav, Anticancer activity of chitosan, chitosan derivatives, and their mechanism of action. 2018. 2018.

34. Unsoy, G., et al., Synthesis of Doxorubicin loaded magnetic chitosan nanoparticles for $\mathrm{pH}$ responsive targeted drug delivery. 2014. 62: p. 243-250.

35. Tiyaboonchai, W.J.N.U.J.S. and Technology, Chitosan nanoparticles: a promising system for drug delivery. 2013. 11(3): p. 51-66.

36. Wang, J.J., et al., Recent advances of chitosan nanoparticles as drug carriers. 2011. 6: p. 765.

37. Min, K.H., et al., Hydrophobically modified glycol chitosan nanoparticles-encapsulated camptothecin enhance the drug stability and tumor targeting in cancer therapy. 2008. 127(3): p. 208-218.

38. Nagpal, K., et al., Chitosan nanoparticles: a promising system in novel drug delivery. 2010. 58(11): p. 1423-1430.

39. Sinha, V., et al., Chitosan microspheres as a potential carrier for drugs. 2004. 274(1-2): p. 1-33.

40. Pan, Y., et al., Bioadhesive polysaccharide in protein delivery system: chitosan nanoparticles improve the intestinal absorption of insulin in vivo. 2002. 249(1-2): p. 139-147.

41. Mitra, S., et al., Tumour targeted delivery of encapsulated dextran-doxorubicin conjugate using chitosan nanoparticles as carrier. 2001. 74(1-3): p. 317-323.

42. Sahu, S.K., et al., In vitro evaluation of folic acid modified carboxymethyl chitosan nanoparticles loaded with doxorubicin for targeted delivery. 2010. 21(5): p. 1587-1597.

43. Yang, S.-J., et al., Folic acid-conjugated chitosan nanoparticles enhanced protoporphyrin IX accumulation in colorectal cancer cells. 2010. 21(4): p. 679-689.

44. Kularatne, S.A. and P.S. Low, Targeting of nanoparticles: folate receptor, in Cancer Nanotechnology. 2010, Springer. p. 249-265.

45. Xu, L., et al., Folate-mediated chemotherapy and diagnostics: an updated review and outlook. 2017. 252: p. 73-82.

46. Kefayat, A., et al., Ultra-small but ultra-effective: Folic acid-targeted gold nanoclusters for enhancement of intracranial glioma tumors' radiation therapy efficacy. 2019. 16: p. 173-184. 
47. Kefayat, A., et al., Investigation of different targeting decorations effect on the radiosensitizing efficacy of albumin-stabilized gold nanoparticles for breast cancer radiation therapy. 2019. 130: $p$. 225-233.

48. O'Shannessy, D.J., et al., Folate receptor alpha (FRA) expression in breast cancer: identification of a new molecular subtype and association with triple negative disease. 2012. 1(1): p. 22.

49. Zhang, Z., et al., Folate receptor a associated with triple-negative breast cancer and poor prognosis. 2014. 138(7): p. 890-895.

50. Necela, B.M., et al., Folate receptor-a (FOLR1) expression and function in triple negative tumors. 2015. 10(3).

51. Paulmurugan, R., et al., Folate receptor-targeted polymeric micellar nanocarriers for delivery of orlistat as a repurposed drug against triple-negative breast cancer. 2016. 15(2): p. 221-231.

52. Kaur, P., et al., A mouse model for triple-negative breast cancer tumor-initiating cells (TNBC-TICS) exhibits similar aggressive phenotype to the human disease. 2012. 12(1): p. 120.

53. Pulaski, B.A. and S.J.C.p.i.i. Ostrand-Rosenberg, Mouse 4T1 breast tumor model. 2000. 39(1): p. 20.2. 1-20.2. 16.

54. Ghahremani, F., et al., AS1411 aptamer conjugated gold nanoclusters as a targeted radiosensitizer for megavoltage radiation therapy of $4 T 1$ breast cancer cells. 2018. 8(8): p. 4249-4258.

55. Ghahremani, F., et al., AS1411 aptamer-targeted gold nanoclusters effect on the enhancement of radiation therapy efficacy in breast tumor-bearing mice. 2018. 13(20): p. 2563-2578.

56. Dehghani, S., et al., Multifunctional MIL-Cur@ FC as a theranostic agent for magnetic resonance imaging and targeting drug delivery: in vitro and in vivo study. 2020. 28(6): p. 668-680.

57. Liu, Y., et al., Novel albendazole-chitosan nanoparticles for intestinal absorption enhancement and hepatic targeting improvement in rats. 2013. 101(6): p. 998-1005.

58. Parakh, D.R., et al., Development and validation of Spectrophotometric method for estimation of mebendazole in bulk and pharmaceutical formulation. 2015. 4: p. 2223-35.

59. Nejadshafiee, V., et al., Magnetic bio-metal-organic framework nanocomposites decorated with folic acid conjugated chitosan as a promising biocompatible targeted theranostic system for cancer treatment. 2019. 99: p. 805-815.

60. Care, O.o.A. and Use, Guidelines for Endpoints in Animal Study Proposals. 2011.

61. Workman, P., et al., Guidelines for the welfare and use of animals in cancer research. 2010. 102(11): p. 1555-1577.

62. Wallace, J., Humane endpoints and cancer research. ILAR journal, 2000. 41(2): p. 87-93.

63. Ibrahim, K., et al., Histopathology of the Liver, Kidney, and Spleen of Mice Exposed to Gold Nanoparticles. 2018. 23(8): p. 1848.

64. Rajeh, M.A.B., et al., Acute toxicity impacts of Euphorbia hirta $L$ extract on behavior, organs body weight index and histopathology of organs of the mice and Artemia salina. 2012. 4(3): p. 170. 
65. Zhang, X.D., et al., Enhanced Tumor Accumulation of Sub-2 $\mathrm{nm}$ Gold Nanoclusters for Cancer Radiation Therapy. 2014. 3(1): p. 133-141.

66. Beidokhti, H.R.N., et al., Preparation, characterization, and optimization of folic acid-chitosanmethotrexate core-shell nanoparticles by box-behnken design for tumor-targeted drug delivery. 2017. 18(1): p. 115-129.

67. Devendiran, R.M., et al., Green synthesis of folic acid-conjugated gold nanoparticles with pectin as reducing/stabilizing agent for cancer theranostics. 2016. 6(35): p. 29757-29768.

68. Esfandiarpour-Boroujeni, S., et al., Fabrication and study of curcumin loaded nanoparticles based on folate-chitosan for breast cancer therapy application. 2017. 168: p. 14-21.

69. Cheng, L., et al., Synthesis of folate-chitosan nanoparticles loaded with ligustrazine to target folate receptor positive cancer cells. 2017. 16(2): p. 1101-1108.

70. Gunasekaran, S. and D.J.A.J.o.C. Uthra, Vibrational spectra and qualitative analysis of albendazole and mebendazole. 2008. 20(8): p. 6310.

71. Souza, T.G., V.S. Ciminelli, and N.D.S. Mohallem. A comparison of TEM and DLS methods to characterize size distribution of ceramic nanoparticles. in Journal of physics: conference series. 2016. IOP Publishing.

72. Song, $\mathrm{H}$. , et al., Folic acid-chitosan conjugated nanoparticles for improving tumor-targeted drug delivery. 2013. 2013.

73. Welch, D.R. and D.R.J.C.r. Hurst, Defining the hallmarks of metastasis. 2019. 79(12): p. 3011-3027.

74. Kurita, K., et al., Enzymatic degradation of $\beta$-chitin: susceptibility and the influence of deacetylation. 2000. 42(1): p. 19-21.

75. Szymańska, E. and K.J.M.d. Winnicka, Stability of chitosan-a challenge for pharmaceutical and biomedical applications. 2015. 13(4): p. 1819-1846.

76. Vårum, K.M., et al., In vitro degradation rates of partially N-acetylated chitosans in human serum. 1997. 299(1-2): p. 99-101.

77. Funkhouser, J.D. and N.N.J.B.e.b. Aronson, Chitinase family GH18: evolutionary insights from the genomic history of a diverse protein family. 2007. 7(1): p. 96.

\section{Figures}




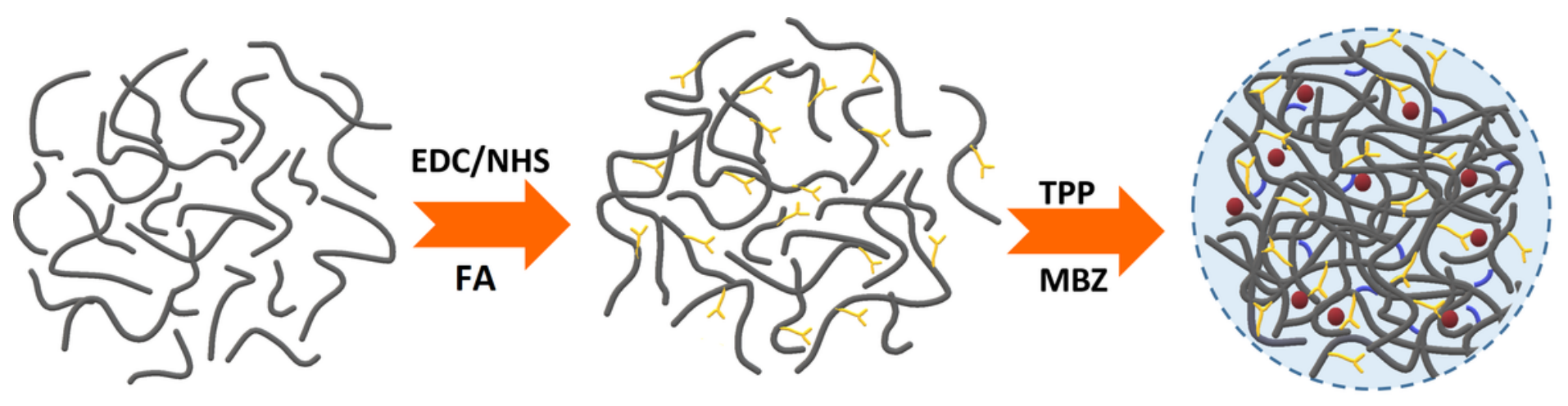

CS
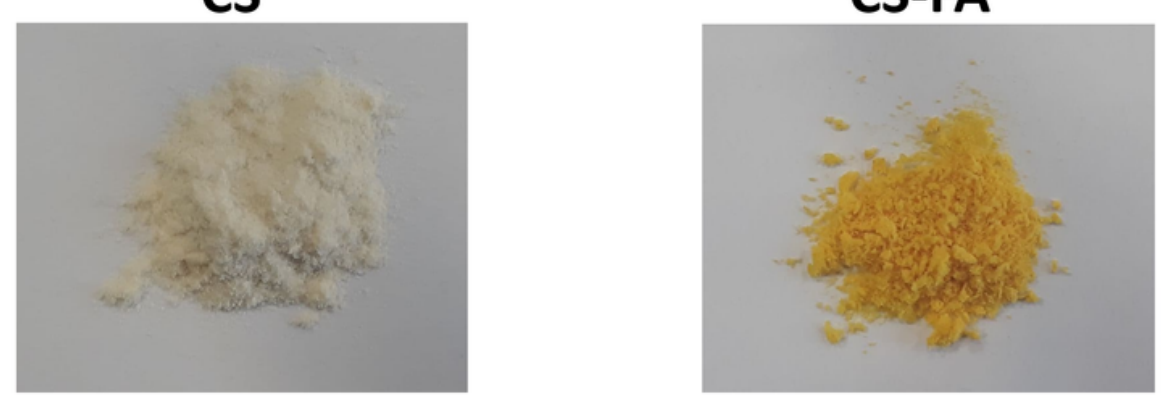

\section{CS-FA-MBZ NPs}

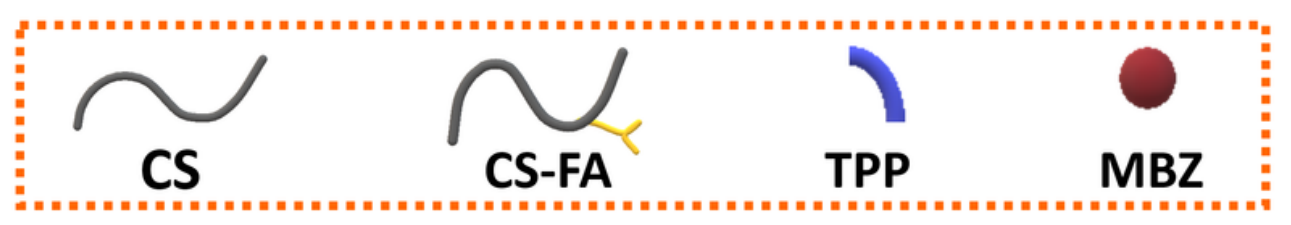

Figure 1

Sequential steps for preparation of the CS-FA-MBZ nanoparticles. 


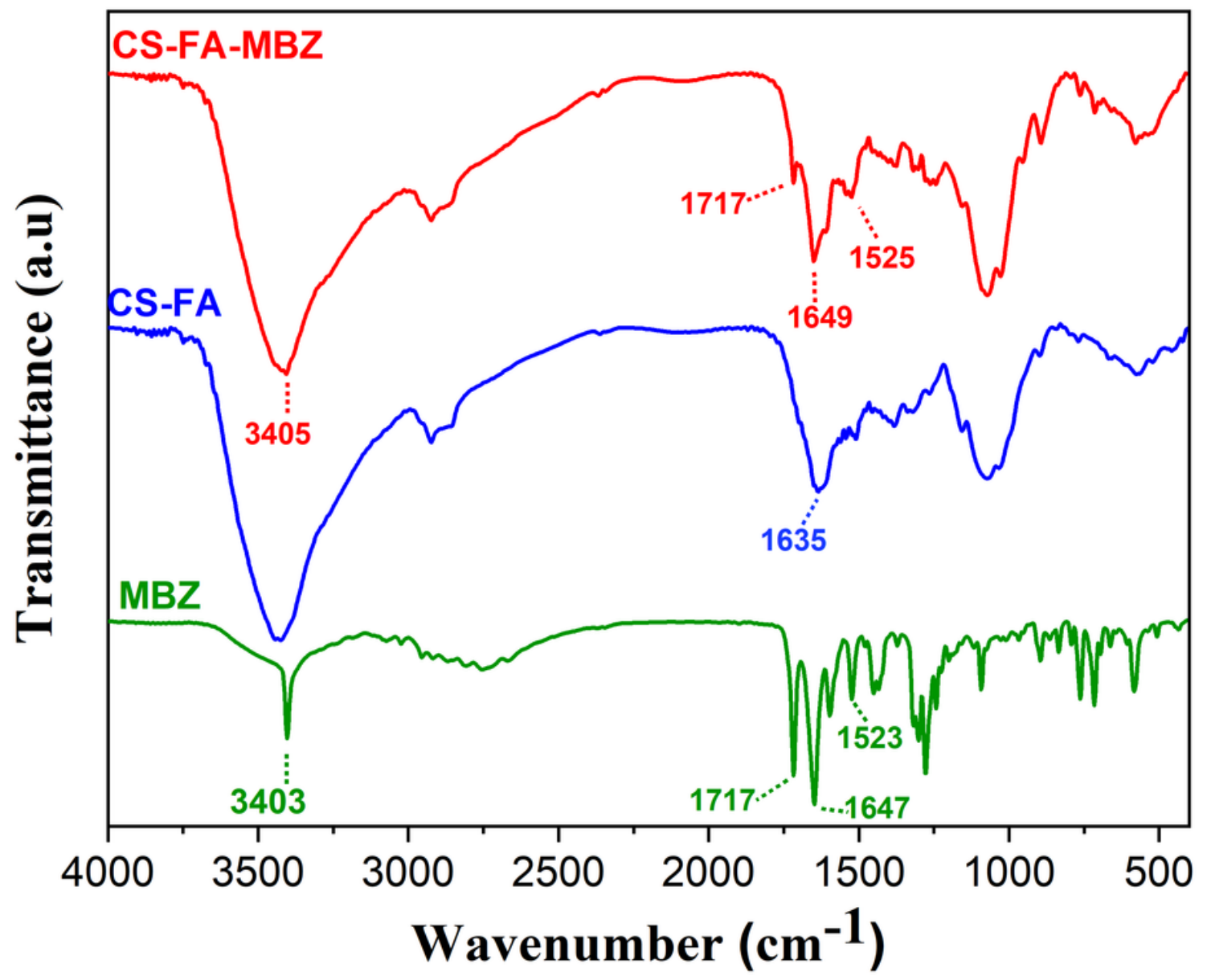

Figure 2

FTIR spectrum of MBZ, CS-FA, and the CS-FA-MBZ nanoparticles. 


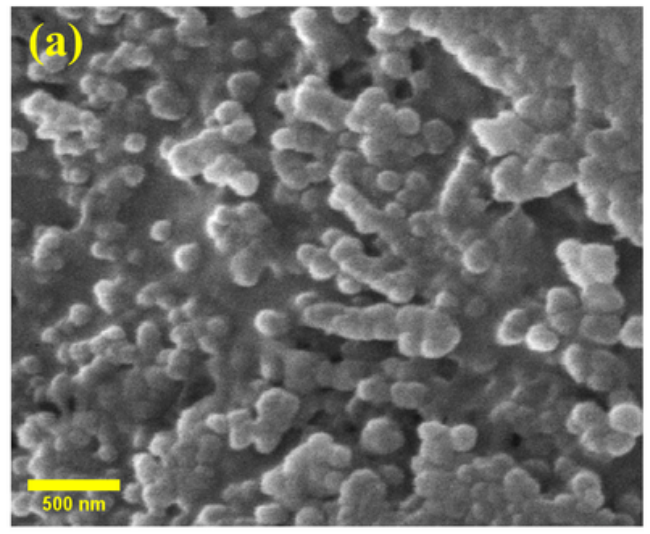

(c)

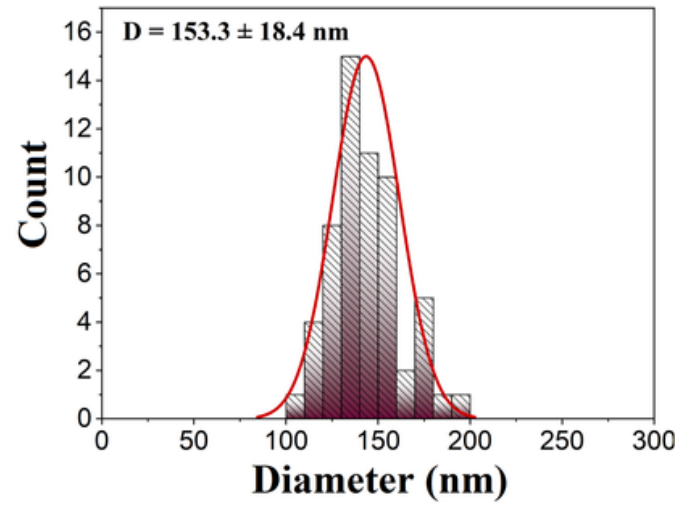

(d)

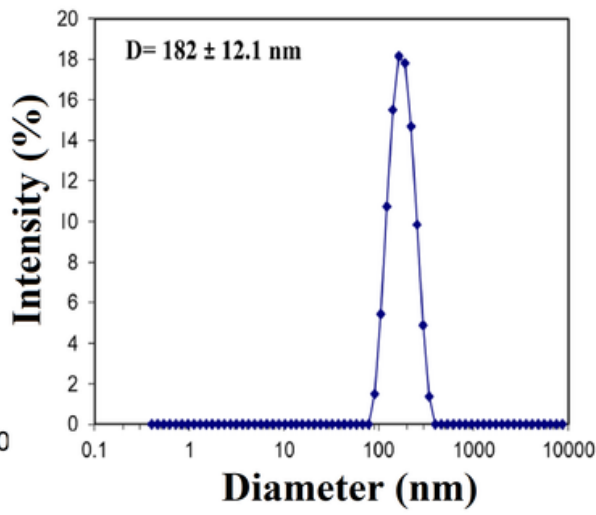

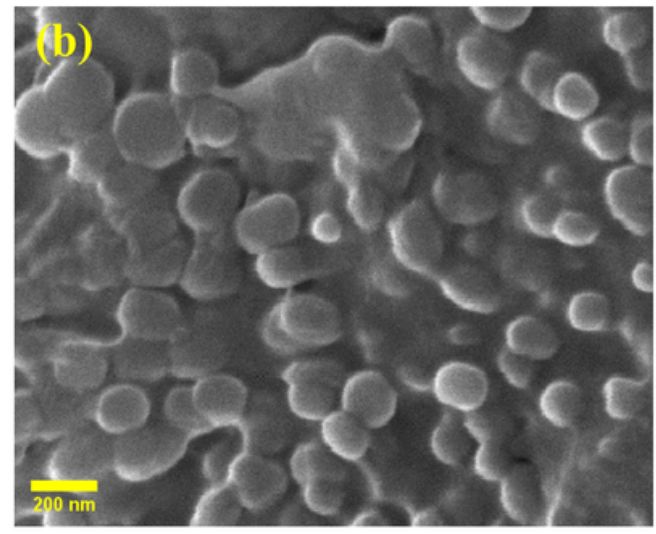

(e)

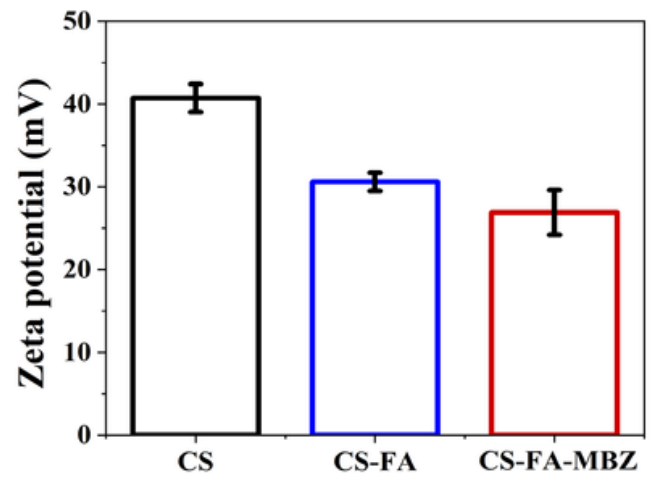

Figure 3

(a, b) SEM images of the CS-FA-MBZ nanoparticles with different magnifications. (c) Size distribution of the CS-FA-MBZ nanoparticles determined by ImageJ software considering 100 particles in corresponding SEM images. (d) The CS-FA-MBZ nanoparticles hydrodynamic size distribution according to DLS measurements. (e) Zeta potential of the Cs, CS-FA, and CS-FA-MBZ nanoparticles.

(a)

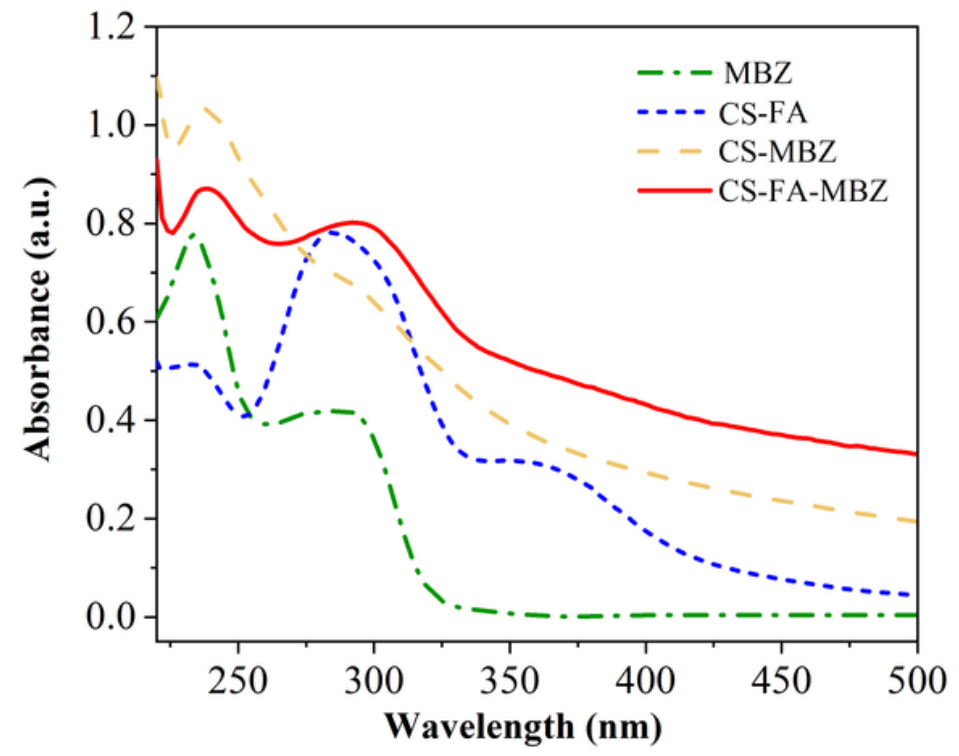

(b)

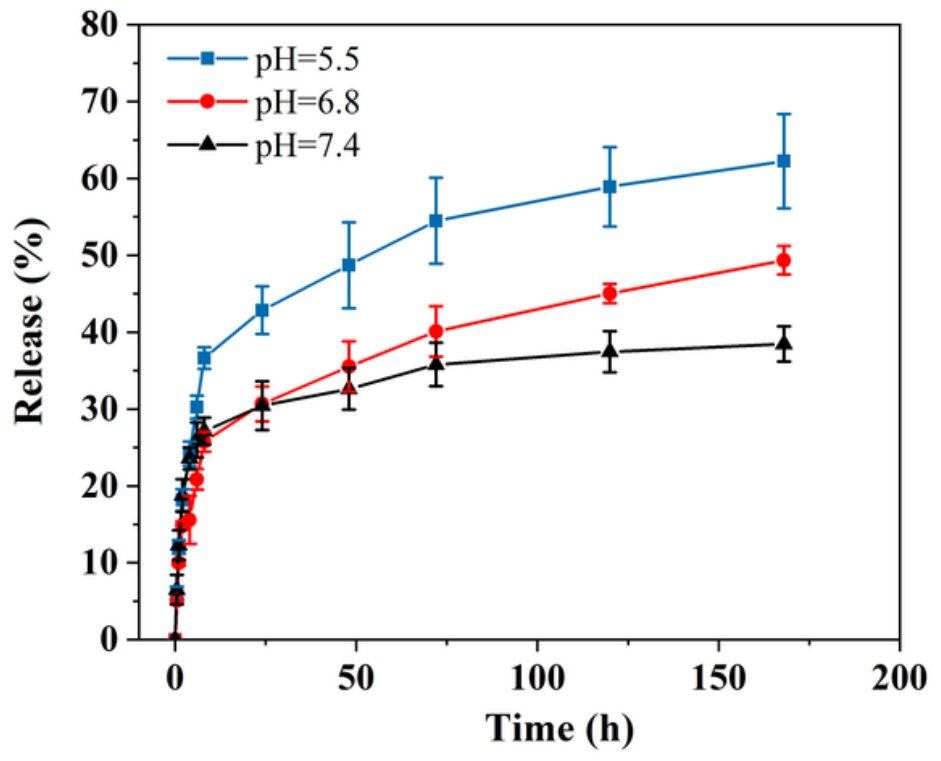

Figure 4 
(a) UV-Vis spectra of MBZ, CS-FA, CS-MBZ, and CS-FA-MBZ. (b) Release profiles of MBZ from CS-FAMBZ nanoparticles at $\mathrm{pH}=5.5,6.8$, and 7.4. Data are expressed as mean \pm S.D $(n=3)$.

(a)

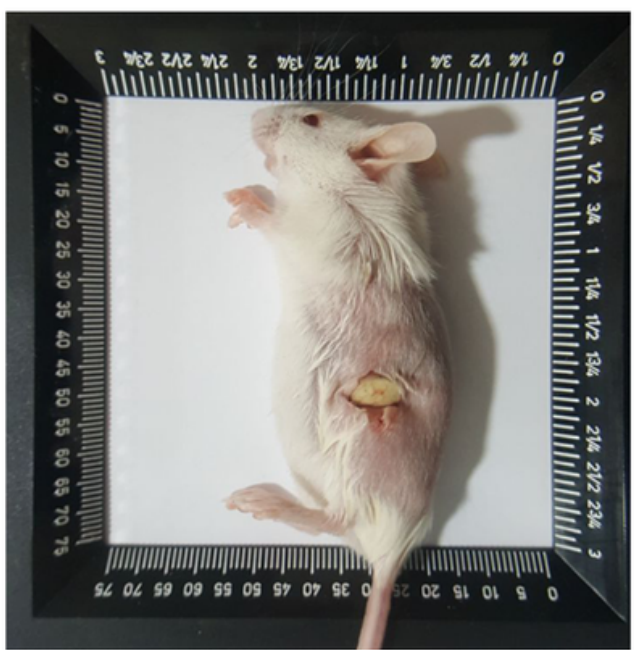

(b)

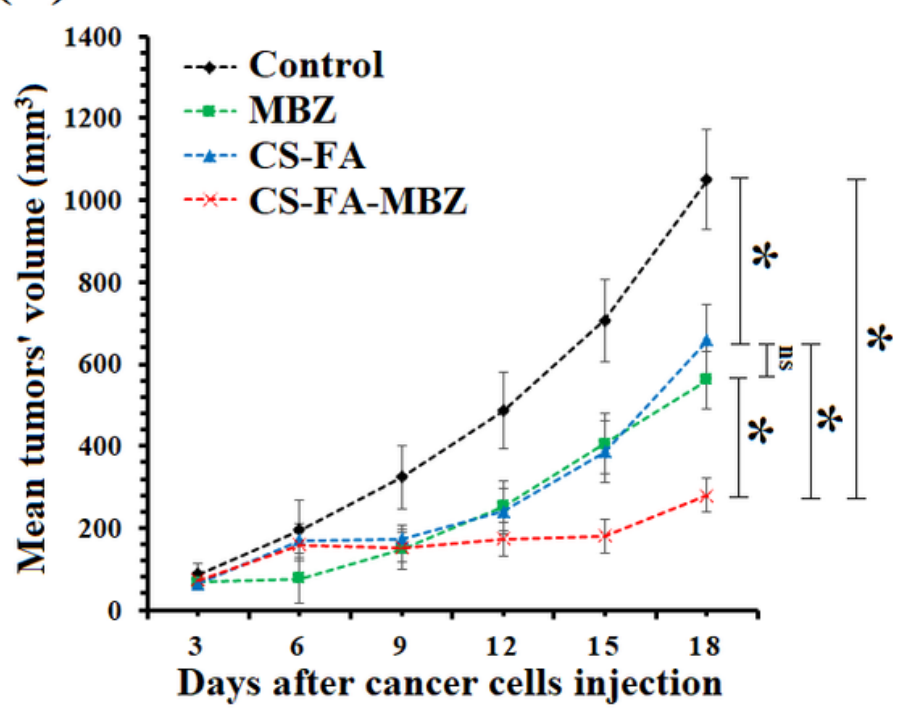

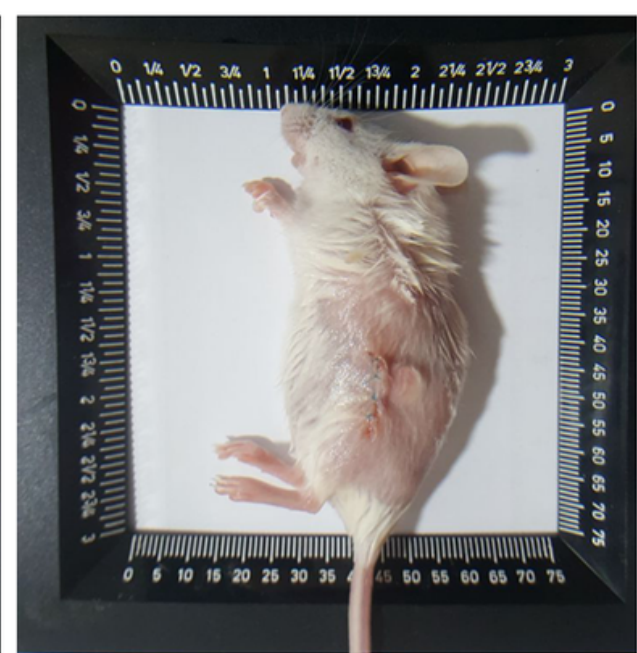

(c)

$$
\text { (c) }
$$

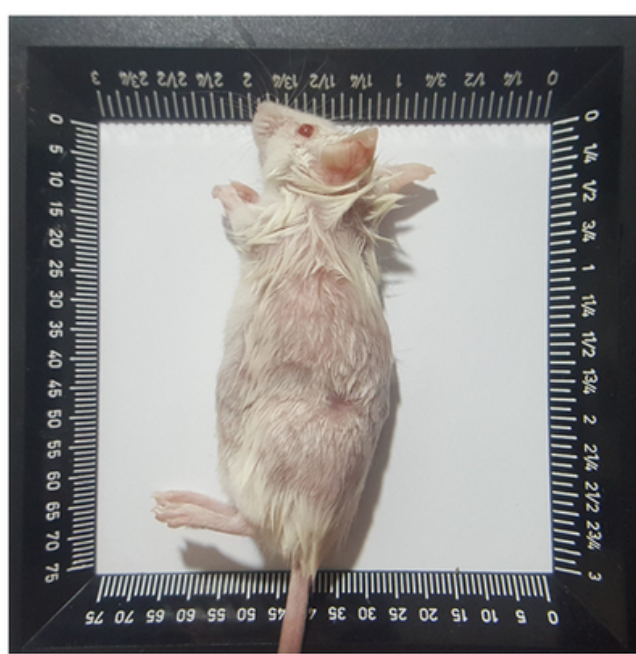

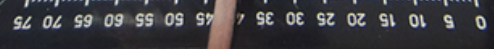

$15^{\text {th }}$ day
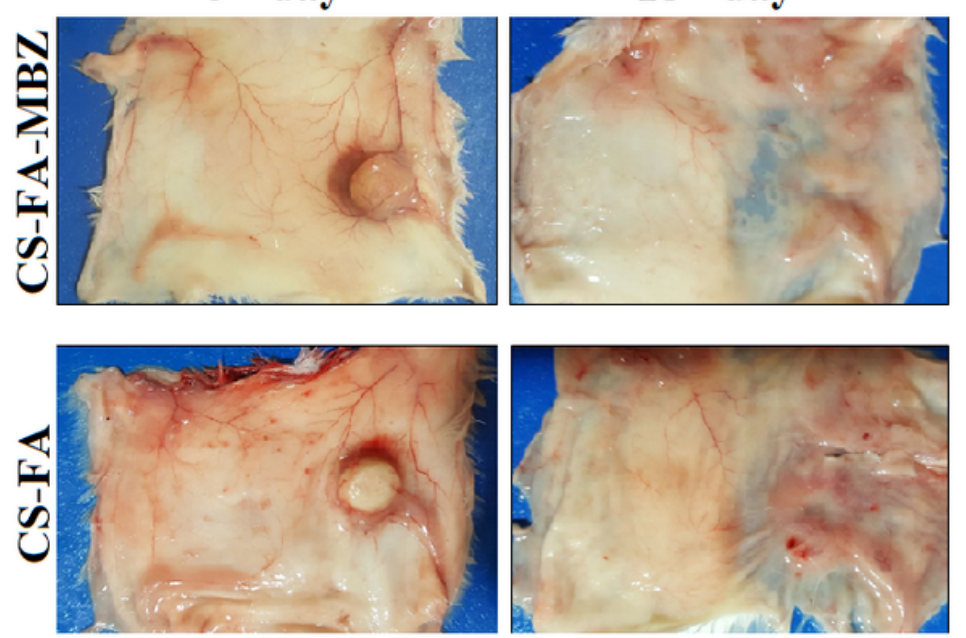

\section{Figure 5}

(a) Subcutaneous implantation of the CS-FA-MBZ implants. A small incision was made at the left flank of mice and the implant was placed inside it. The incision was sutured. The implantation site on the 18th day after cancer cells injection (15th day after surgery). (b) Tumors' growth progression in different groups including Control, MBZ, CS-FA, and CS-FA-MBZ from the 3rd to 18th day after cancer cells injection (not significant: $n s, *$ : $\mathrm{P}<0.05$ ). (c) Harvested skin of the implants-bearing mice at the $3 \mathrm{rd}$ and 15th days after implantation which equates the 6 th and 18th days after cancer cells injection, respectively. 

(a)
(b)

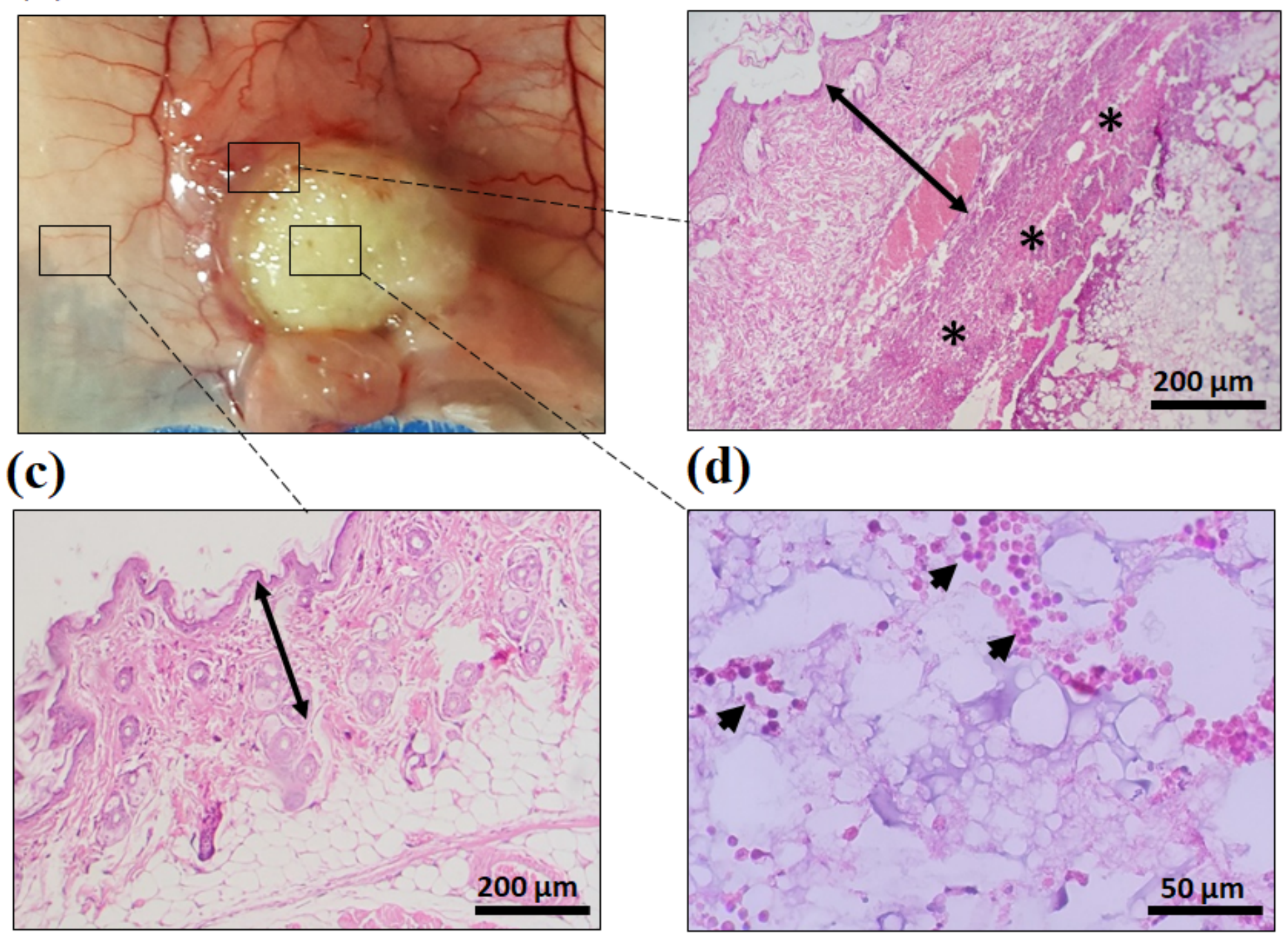

\section{Figure 6}

Mouse body response to the subcutaneously implanted CS-FA-MBZ at 3rd day after implantation according to histopathology analysis. (a) A close-up view of a CS-FA-MBZ implant 3rd day after implantation and histopathological evaluation of (b) the implant-surrounding membrane, (c) tissues far away from the implantation site, and (d) tissues at the bed of the implantation site. The two head arrows, asterisk, and one-head arrows indicate derma, the implant's surrounding membrane, and infiltrating immune cells, respectively. 
(a)

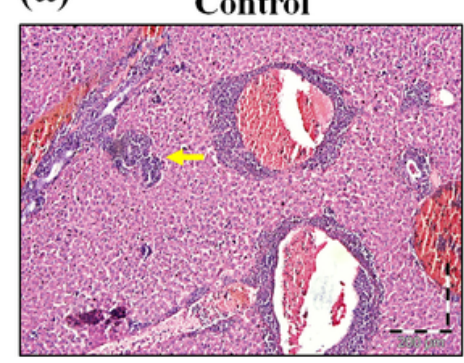

(b)

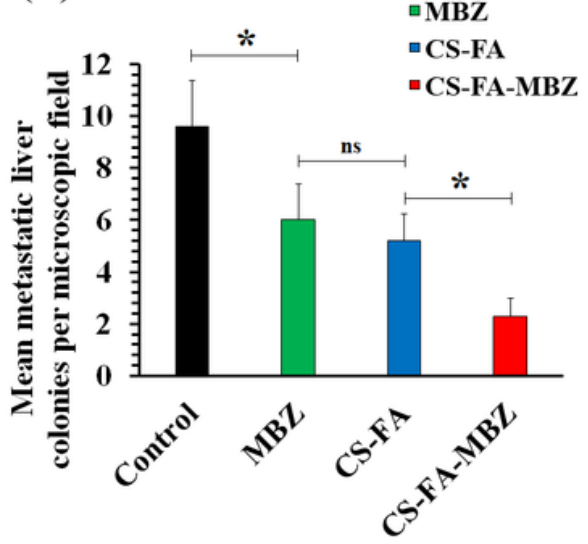

MBZ

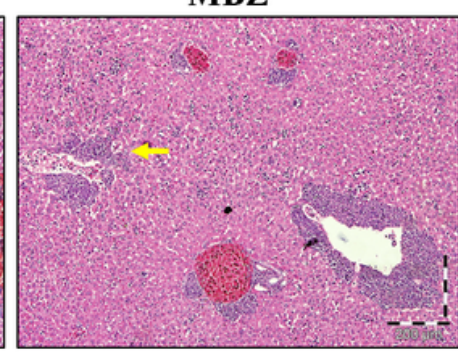

(c)
CS-FA

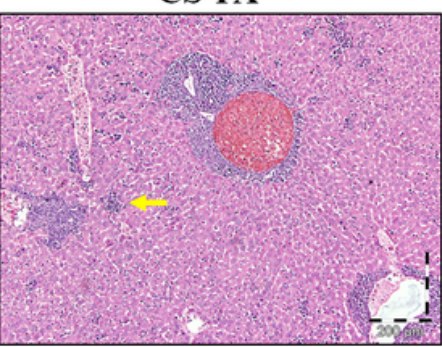

(d)

- Control

$\square$ MBZ

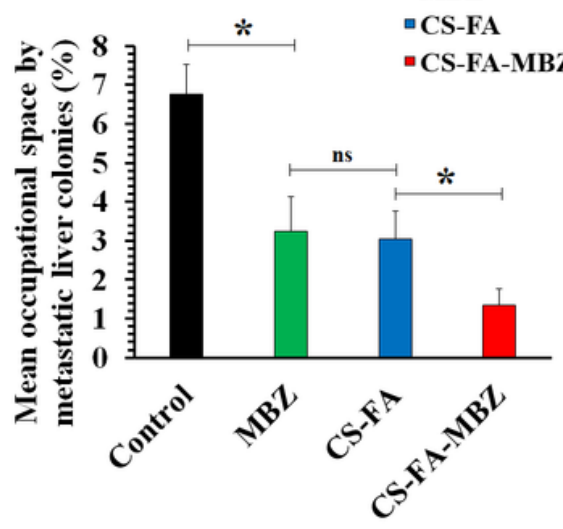

CS-FA-MBZ

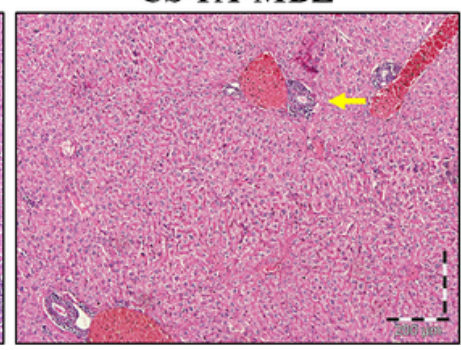

- Control

- MBZ

- CS-FA

- CS-FA-MBZ

\section{Figure 7}

Metastatic burden and survival time of the tumor-bearing mice at different treatment groups. (a) One microscopic field of H\&E stained sections of livers in each group was illustrated as the sample. (b) The average number of metastasis colonies per microscopic field of the tumor-bearing mice's liver 30 days after the cancer cells injection. The yellow arrows indicate a liver metastatic colony. (c) Mean percentage of the occupied space per microscopic field of the tumor-bearing mice's liver 30 days after the cancer cells injection. (d) The survival time of tumor-bearing mice in different groups. (not significant: ns, *: $\mathrm{P}$ $<0.05)$ 
(a)
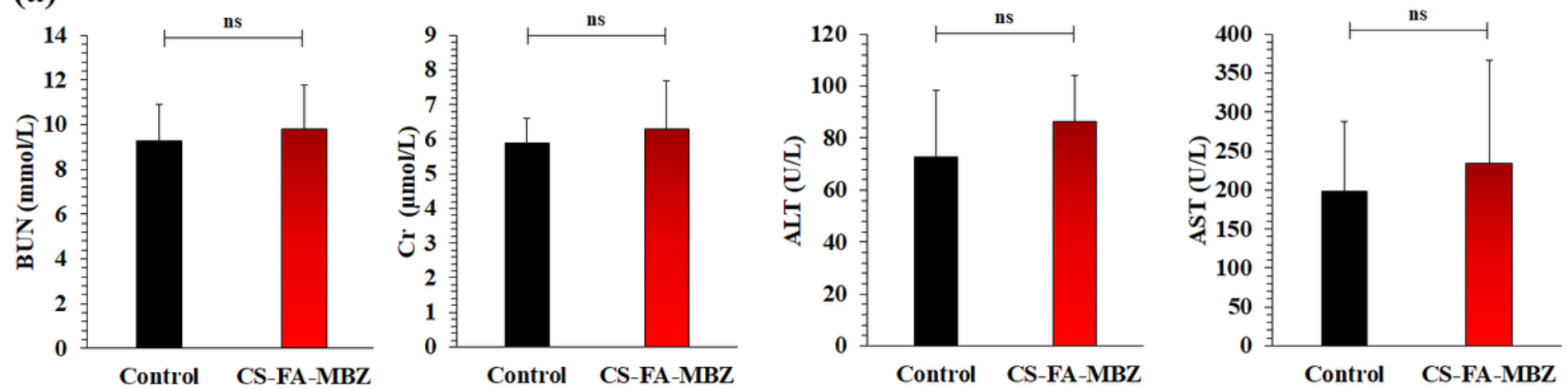

(b)

Lung

Kidney

Liver

Spleen
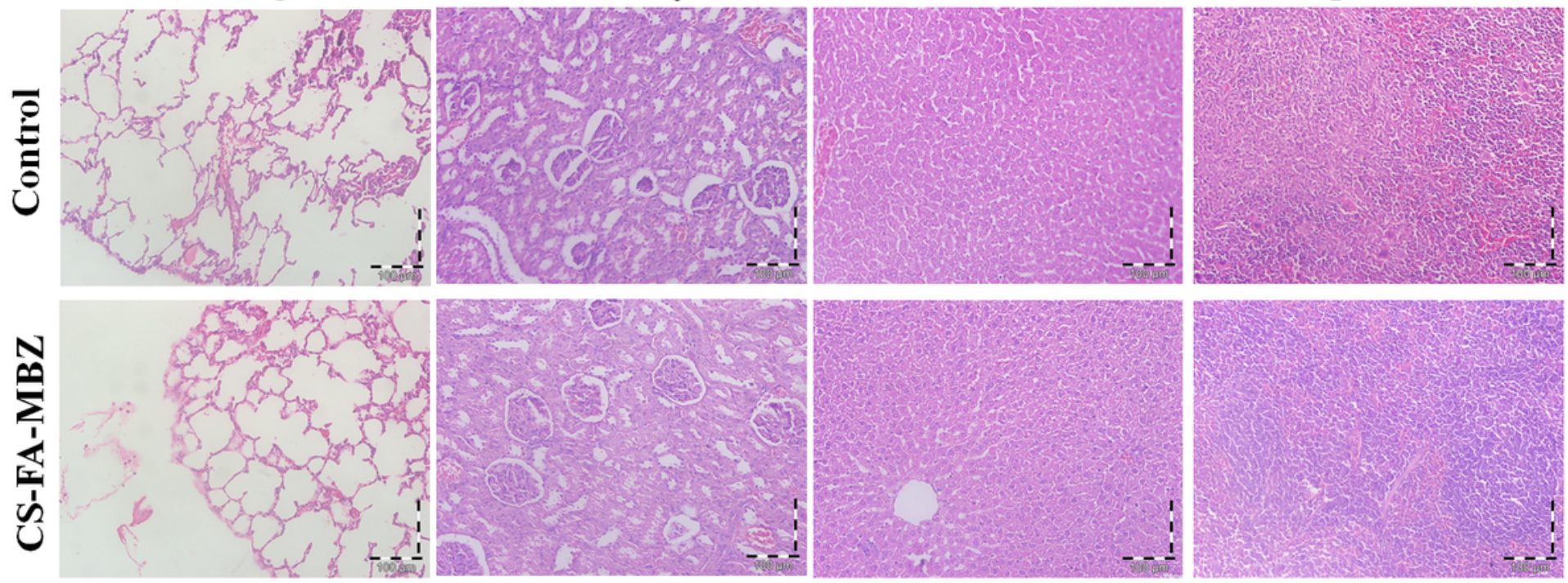

Figure 8

Assessment of the CS-FA-MBZ implants according to blood biochemical analyzes and histopathological evaluation of vital organs. (a) Serum level of BUN, Cr, ALT, and AST of the CS-FA-MBZ implanted mice $(n=5) 30$ days after implantation in comparison with the Control (ns: not significant). (b) H\&E stained sections of lungs, kidneys, spleen, and liver of the CS-FA-MBZ implanted mice $(n=5) 30$ days after implantation in comparison with the Control.

\section{Supplementary Files}

This is a list of supplementary files associated with this preprint. Click to download.

- Graphicalabstract.png

- SupplementaryMaterial.docx 